\title{
Efeito modulador do óleo de copaíba (Copaifera officinalis L.) sobre a carcinogenicidade da doxorrubicina, avaliado por meio do teste para detecção de clones de tumores em Drosophila melanogaster
}

\author{
Modulatory effect of the copaiba oil (Copaifera officinalis L.) on the carcinogenicity of \\ doxorubicin, evaluated through the test for detection of tumor clones in Drosophila \\ melanogaster
}

\author{
Álisson Duarte Maciel ${ }^{1}$, Monique Danielle Magalhães ${ }^{1}$, Priscila Capelari Orsolin ${ }^{2 *}$ \\ ${ }^{1}$ Acadêmico de Medicina. Centro Universitário de Patos de Minas; ${ }^{1}$ Acadêmica de Medicina. Centro Universitário de \\ Patos de Minas; ${ }^{2}$ Doutora em Genética e Bioquímica. Centro Universitário de Patos de Minas
}

\begin{abstract}
Resumo
Introdução: o óleo de copaíba é o composto extraído do tronco da copaibeira (Copaifera sp.) que tem sido utilizado na medicina popular desde a chegada dos portugueses ao Brasil. Objetivo: o objetivo deste estudo foi avaliar possíveis efeitos carcinogênicos e/ ou anticarcinogênicos do óleo de copaíba (Copaifera officinalis L.), por meio do teste para detecção de clones de tumores epiteliais (warts) em Drosophila melanogaster. Metodologia: foram preparadas três soluções de óleo de copaíba, nas proporções de $0,5 \%$, $1 \%$ e $2 \%$. Nessas soluções foram cultivadas Drosophilas melanogaster expostas simultaneamente à doxorrubicina na concentração de $0,4 \mathrm{mM}$, agente conhecidamente cancerígeno, também utilizado como controle positivo na presente pesquisa. Para controle negativo foi utilizado Tween 80 (1\%). O tratamento foi realizado com todas as larvas descendentes do cruzamento de fêmeas wts/ TM3 com machos $\mathrm{mwh} / \mathrm{mwh}$. Resultados: o óleo de copaíba apresentou atividade carcinogênica quando utilizado isoladamente na concentração $2 \%$, visto que houve aumento estatisticamente significativo $(p \leq 0,05)$ na frequência de tumores em comparação com o controle negativo. Além disso, evidenciou-se potencialização do efeito carcinogênico da doxorrubicina nas concentrações $0,5 \%$ e $1 \%$, uma vez que houve um aumento estatisticamente significativo $(p \leq 0,05)$ na frequência de tumores nessas concentrações, quando associadas à DXR, em comparação com o controle positivo. Conclusão: os resultados evidenciaram o efeito carcinogênico isolado do óleo de copaíba, bem como seu efeito potencializador quando associado à doxorrubicina.

Palavras-chave: Plantas Medicinais. Drosophila melanogaster. Testes de Carcinogenicidade. Doxorrubicina.
\end{abstract}

\begin{abstract}
Introduction: the copaiba oil is a compound extracted from the trunk of the copaiba tree (Copaifera sp.) that has been used in popular medicine since the arrival of the Portuguese in Brazil. Purpose: this study aimed to evaluate the possible carcinogenic and/ or anticarcinogenic effects of the copaiba oil (Copaifera officinalis L.) through the test for detection of epithelial tumor clones (warts) in Drosophila melanogaster. Methodology: three solutions of copaiba oil were prepared in the proportions 0,5\%, $1 \%$ and $2 \%$. In these solutions were cultivated Drosophilas melanogaster previously exposed to doxorubicin at a $0.4 \mathrm{mM}$ concentration, admittedly carcinogenic agent, which was also used for positive control in the present research. For negative control Tween 80 (1\%) was used. The treatment was performed on all larvae descendant of the crossing of females wts/TM3 with males mwh/mwh. Results: the results showed that the copaiba oil presented carcinogenic activity when used in isolation at the concentration of $2 \%$, seeing that there was a statistically significant increase $(p \leq 0,05)$ in tumor frequency in comparison to the negative control. Moreover, there was a potentiation of doxorubicin's carcinogenic effect at concentrations $0,5 \%$ and $1 \%$, since there was a statistically significant increase ( $p$ $\leq 0,05)$ of tumor frequency in these concentrations, when associated to DXR, in comparison to the positive control. Conclusion: the results evidenced the isolated carcinogenic effect of copaiba oil, as well as its potentiating effect when associated with doxorubicin. Keywords: Plants, Medicinal. Drosophila melanogaster. Carcinogenicity Tests. Doxorubicin.
\end{abstract}

\section{INTRODUÇÃO}

As plantas com propriedades medicinais são importantes não somente por seu uso popular, mas também para a pesquisa farmacológica e o desenvolvimento de drogas, podendo ser utilizadas diretamente como

Correspondente/Corresponding: Priscila Capelari Orsolin - End: Rua Major Gote, número 808, Bairro Caiçaras; Laboratório de Citogenética e Mutagênese (bloco M). Patos de Minas -MG. - Tel: (34) 3823-0169 E-mail: pricaorsolin@hotmail.com agentes terapêuticos, como matérias-primas para a síntese ou como modelos para compostos farmacologicamente ativos (BRASIL, 2006). Aproximadamente $40 \%$ dos medicamentos disponíveis em 2001 eram derivados direta ou indiretamente de fontes naturais, sendo que as plantas representavam $25 \%$ dessa parcela (CALIXTO et al., 2001).

Embora o Brasil possua a maior diversidade vegetal do planeta (GIULIETTI et al., 2005), segundo Guerra e 
Nodari (2001), apenas $8 \%$, cerca de cinco mil espécies, foram estudadas para pesquisas de compostos bioativos e, representando uma parcela ainda menor, apenas 1.100 tiveram suas propriedades medicinais avaliadas. Percebe-se, assim, um subaproveitamento do potencial de desenvolvimento de fitoterápicos e até mesmo novos fármacos no país.

Atualmente, um dos grandes desafios da medicina é o tratamento dos cânceres, que pode ser feito através de cirurgia, radioterapia, quimioterapia ou transplante de medula óssea, a depender da localização, extensão e o tipo de câncer. Apesar dos métodos disponíveis e o constante avanço da medicina tradicional, observa-se cada vez mais a procura por tratamentos alternativos ou complementares, tendo foco o uso de espécies e extratos vegetais, dentre os quais podemos citar o óleo de copaíba (OLIVEIRA; MACHADO; RODRIGUES, 2014).

O gênero Copaifera é representado pelas copaibeiras e possui 72 espécies descritas, sendo 16 delas encontradas exclusivamente no Brasil (VEIGA JUNIOR; PINTO, 2002). Tratam-se de árvores de grande porte, que podem atingir entre 30 e 40 metros de altura e diâmetro entre 0,4 e quatro metros (ARAÚJO JÚNIOR et al., 2005).

O óleo de copaíba é composto de uma parte sólida diluída em outro componente, um óleo essencial. A primeira porção corresponde de $55 \%$ a $60 \%$ do óleo e é composto por ácidos diterpênicos. Já o óleo essencial é composto basicamente por sesquiterpenos (RIGAMONTE-AZEVEDO; WADT; WADT, 2004). Apesar de poderem ocorrer variações nas proporções das substâncias e os tipos de diterpenos e sesquiterpenos presentes no óleo de copaíba, estas são as únicas classes de substâncias que podem ser encontradas no óleo sem alterações (VEIGA JUNIOR; PINTO; MACIEL, 2005).

Os principais sesquiterpenos encontrados no óleo de copaíba são $\beta$-cariophileno, que possui comprovada ação anti-inflamatória, antibacteriana e antifúngica e $\beta$-bisaboleno, que possui propriedades anti-inflamatórias e analgésicas (OLIVEIRA; LAMEIRA; ZOGHBI, 2006; VEIGA JUNIOR; PINTO, 2002).

Pela frequente indicação do óleo no uso tradicional, o mesmo tem sido, há algum tempo, matéria de vários estudos com objetivo de comprovar as propriedades a ele atribuídas. Com o avanço dessas pesquisas já foram evidenciadas atividades anti-inflamatória, gastroprotetora, analgésica e antitumoral (BASILE et al., 1988; FERNANDES; PEREIRA; PAULO, 1992; OHSAKI et al., 1994; PAIVA et al., 1998). Efeitos cicatrizante (MASSON, 2011), bactericida e bacteriostático (DRUMOND et al., 2004), antisséptico e antiviral (VEIGA JUNIOR; PINTO, 2002), também são citados.

Não há relatos de toxicidade do óleo de copaíba quando administrado por via oral e em aplicações cutâneas. No entanto, Costa-Lotufo et al. (2002) observaram que o óleo pode possuir embriotoxicidade, e por não haver estudos conclusivos, o uso oral durante a gestação deve ser evitado.
Apesar de existirem evidências, os trabalhos realizados a fim de se avaliar as propriedades medicinais do óleo de copaíba têm-se apresentado ainda restritos. Novas pesquisas devem ser realizadas, direcionadas principalmente a importantes atividades descritas, como antitumoral, anti-inflamatória e antimicrobiana (PIERI; MUSSI; MOREIRA, 2009).

Diante do exposto, o presente trabalho teve como objetivo avaliar se o óleo copaíba (Copaifera officinalis L.) apresenta efeito anticarcinogênico ou mesmo carcinogênico, por meio do teste para detecção de clones de tumores epiteliais (warts) em Drosophila melanogaster, a fim de complementar as informações obtidas até o momento pela comunidade científica acerca do assunto.

\section{METODOLOGIA}

agente biológico - óleo de copaíba (Copaifera officinalis L.)

Para uso popular, o óleo de copaíba pode ser adquirido já envasado por empresas que o comercializam ou colhido diretamente do tronco da copaibeira. Neste trabaIho optou-se pela primeira opção. O óleo foi adquirido em apresentação de frasco contendo $50 \mathrm{~mL}$, sendo a espécie de origem a Copaifera officinalis L. Produto extraído por Distriol - Extração Vegetal Ltda., envasado por Real Nutry Industria e Comercio Ltda. e distribuído por ACQ \& GBM Comércio e Desenvolvimento Ltda - lote COP680-1/7229. Produto dispensado de Registro - resolução ANVISA - RDC 27, de 06 de agosto de 2010 - Anexo I - Item 4100158.

agente químico - Doxorrubicina (DXR)

Como agente indutor de tumor e controle positivo foi utilizada a doxorrubicina (CAS 23214-92-8), comercializada como Adriblastina ${ }^{\circledR}$ RD. A mesma é produzida pelo laboratório Pfizer sob a forma de pó liofilizado injetável de $50 \mathrm{mg}$ em embalagem contendo um frasco-ampola (Farmacêutico responsável José Cláudio Bumerad. CRF-SP $n^{\circ}$ 437146). O referido composto foi utilizado na concentração de 0,4 mM, utilizando Tween 80 (1\%) como solvente para preparação.

\section{TESTE PARA DETECÇÃO DE TUMORES EPITELIAIS EM Drosophila melanogaster}

Para realização do teste foram utilizadas duas linhagens mutantes de Drosophila melanogaster, wts e mwh, portadoras dos marcadores genéticos warts (wts, 3-100) e multiple wing hairs, (3-03). A linhagem wts foi fornecida pelo Bloomington Drosophila Stock Center, da Universidade de Indiana nos Estados Unidos (USA), registrado sob o número: Bloomington/7052. Já a linhagem mwh/ mwh foi cedida pelo Dr. Ulrich Graf (Physiology and Animal Husbandry, Institute of Animal Science, ETH Zurich, Schwerzenbach, Switzerland).

Os estoques destas linhagens são cultivados no Laboratório de Citogenética e Mutagênese do Centro Universitário de Patos de Minas - UNIPAM, mantidas em frascos de $1 / 4$ de litro contendo o meio de cultura da Drosophila melanogaster. Esse meio é composto por $820 \mathrm{~mL}$ de água, 
$25 \mathrm{~g}$ de fermento (Sacchoromyces cerevisae), $11 \mathrm{~g}$ de ágar, $156 \mathrm{~g}$ de banana e um grama de nipagin. As linhagens são conservadas dentro de uma incubadora B.O.D. 411 D, a uma temperatura em torno de $25^{\circ} \mathrm{C}$ e $60 \%$ de umidade.

\section{Cruzamento}

Para a realização dos cruzamentos, machos e fêmeas foram acondicionados em frascos contendo meio de cultura próprio para postura, onde as fêmeas depositaram seus ovos após acasalamento. Para obtenção de larvas heterozigotas $w t s+/+m w h$ foi realizado o cruzamento entre fêmeas virgens wts/TM3,S $b^{1}$ com machos $m w h /$ $m w h$. As larvas descendentes deste cruzamento foram tratadas com óleo de copaíba em três diferentes concentrações (0,5\%, $1 \%$ e $2 \%)$, associadas e não associadas à doxorrubicina.

A coleta dos ovos descendentes dos cruzamentos entre fêmeas virgens wts/TM3, Sb ${ }^{1}$ com machos $m w h / m w h$, ocorreu durante um período de oito horas, em frascos contendo meio de cultura próprio para postura, uma base sólida de ágar ( $3 \%$ de ágar em água) e uma camada de fermento biológico suplementado com sacarose.

\section{Tratamento}

Larvas de terceiro estágio foram submetidas a um tratamento crônico, por um período de aproximadamente 48 horas (quando sobem às paredes dos frascos). Estas larvas foram colocadas em frascos de vidro de $25 \mathrm{~mL}$, contendo $1,5 \mathrm{~g}$ de purê de batata e diferentes concentrações e combinações de óleo de copaíba, controle positivo e negativo. Como controle positivo foi utilizada a doxorrubicina $(0,4$ $\mathrm{mM}$ ) e como controle negativo Tween 80 (1\%). Sendo assim, foram montados os seguintes frascos:

1. Purê de batata $(1,5 \mathrm{~g})+$ Tween $80(1 \%)+$ larvas $=$ controle negativo

2. Purê de batata $(1,5 \mathrm{~g})+\operatorname{DXR}(0,4 \mathrm{mM})+$ larvas $=$ controle positivo

3. Purê de batata $(1,5 \mathrm{~g})+$ óleo de copaíba $(0,5 \%)$ + larvas

4. Purê de batata $(1,5 \mathrm{~g})+$ óleo de copaíba $(1 \%)+$ larvas

5. Purê de batata $(1,5 \mathrm{~g})+$ óleo de copaíba $(2 \%)+$ larvas

6. Purê de batata $(1,5 \mathrm{~g})+\operatorname{DXR}(0,4 \mathrm{mM})+$ óleo de copaíba $(0,5 \%)+$ larvas

7. Purê de batata $(1,5 \mathrm{~g})+\mathrm{DXR}(0,4 \mathrm{mM})+$ óleo de copaíba (1\%) + larvas

8. Purê de batata $(1,5 \mathrm{~g})+\operatorname{DXR}(0,4 \mathrm{mM})+$ óleo de copaíba (2\%) + larvas

\section{Análise das moscas}

Após o tratamento, todas as moscas foram coletadas e armazenadas em frascos devidamente identificados, contendo etanol $70 \%$ (para conservação). Posteriormente, elas foram colocadas individualmente numa placa escavada contendo glicerina e analisadas numa lupa estereoscópica para visualização e contagem dos tumores, de acordo com a descrição de Justice et al. (1995), com auxílio de um pincel para manusear as moscas.

Embora todas as moscas tenham sido coletadas, somente as moscas adultas de pelos longos e finos foram analisadas, ou seja, somente as moscas portadoras do gene wts. As moscas adultas que apresentavam pelos curtos e grossos foram descartadas, uma vez que não possuem o gene em estudo.

O registro da frequência de tumores foi realizado em planilha padrão, que separa quantitativamente o número de tumores nas regiões do olho, cabeça, asas, corpo, pernas, halteres e o total de tumores por mosca, em cada concentração testada.

\section{ANÁLISE ESTATÍSTICA}

As diferenças estatísticas entre a frequência de tumores das concentrações testadas e os controles (positivo e negativo) foram calculadas utilizando o teste $U$, não paramétrico, de Mann-Whitney, empregando o nível de significância de $5 \%$.

\section{RESULTADOS}

Através da Tabela 1 é possível verificar a ocorrência e a frequência de tumores nos diferentes segmentos corporais da Drosophila melanogaster. Para controle negativo foi utilizado Tween 80 (1\%), que demonstrou uma frequência de 0,19 tumores por mosca ou 37 tumores em um total de 200 moscas analisadas, em números absolutos. Segundo Alves e Nepomuceno (2012), essa discreta indução de tumores ocorre devido à predisposição genética intrínseca da Drosophila melanogaster. De modo contrário, as moscas tratadas com doxorrubicina $(0,4 \mathrm{mM})$ (controle positivo) apresentaram frequência de 1,10 tumor por mosca, mostrando que a linhagem é suscetível à indução tumoral.

Tabela 1-Frequência de clones de tumores observados em Drosophila melanogaster, heterozigota para o gene supressor de tumor wts, tratada com doxorrubicina (controle positivo), Tween 80, 1\% (controle negativo) e diferentes concentrações de óleo de copaíba.

\begin{tabular}{|c|c|c|c|c|c|c|c|c|c|c|}
\hline \multirow{2}{*}{$\begin{array}{l}\text { Tratamentos } \\
\text { de Copaíba } \\
(\%)\end{array}$} & \multirow[b]{2}{*}{$\begin{array}{l}\text { DXR } \\
(\mathrm{mM})\end{array}$} & \multirow[b]{2}{*}{$\begin{array}{c}\text { Número de moscas } \\
\text { analisadas }\end{array}$} & \multicolumn{7}{|c|}{ Número de tumores analisados } & \multirow[b]{2}{*}{$\begin{array}{c}\text { Frequência (No de } \\
\text { tumores/ mosca) }\end{array}$} \\
\hline & & & Olho & Cabeça & Asa & Corpo & Perna & Halter & Total & \\
\hline 0 & 0 & 200 & 0 & 3 & 9 & 12 & 10 & 3 & 37 & 0,19 \\
\hline 0 & 0,4 & 200 & 3 & 19 & 104 & 25 & 28 & 32 & 211 & $1,10 *$ \\
\hline 0,5 & 0 & 200 & 0 & 8 & 9 & 20 & 4 & 6 & 47 & $0,24 \mathrm{~ns}^{*}$ \\
\hline
\end{tabular}


Álisson Duarte Maciel, Monique Danielle Magalhães, Priscila Capelari Orsolin

\begin{tabular}{|c|c|c|c|c|c|c|c|c|c|c|}
\hline \multirow{2}{*}{$\begin{array}{l}\text { Tratamentos } \\
\text { de Copaíba } \\
(\%)\end{array}$} & \multirow[b]{2}{*}{$\begin{array}{l}\text { DXR } \\
(\mathrm{mM})\end{array}$} & \multirow[b]{2}{*}{$\begin{array}{c}\text { Número de moscas } \\
\text { analisadas }\end{array}$} & \multicolumn{7}{|c|}{ Número de tumores analisados } & \multirow[b]{2}{*}{$\begin{array}{c}\text { Frequência (No de } \\
\text { tumores/ mosca) }\end{array}$} \\
\hline & & & Olho & Cabeça & Asa & Corpo & Perna & Halter & Total & \\
\hline 1 & 0 & 200 & 0 & 6 & 6 & 10 & 2 & 20 & 44 & 0,22 ns* $^{*}$ \\
\hline 2 & 0 & 200 & 0 & 11 & 6 & 22 & 6 & 6 & 51 & $0,26 *$ \\
\hline 0,5 & 0,4 & 200 & 2 & 38 & 130 & 22 & 104 & 129 & 425 & $2,13 * *$ \\
\hline 1 & 0,4 & 200 & 2 & 54 & 166 & 24 & 70 & 72 & 388 & $1,94 * *$ \\
\hline 2 & 0,4 & 200 & 1 & 18 & 86 & 25 & 61 & 57 & 248 & $1,24 \mathrm{~ns}^{* *}$ \\
\hline
\end{tabular}

Diagnóstico estatístico de acordo com o Teste de Mann-Whitney. Nível de significância $p \leq 0,05$.

* Valor considerado diferente do controle negativo $(p \leq 0,05)$.

** Valor considerado diferente do controle positivo (DXR 0,4 mM) ( $p \leq 0.05)$.

$n s^{*} \quad$ valores considerados não significativos quando comparados com o controle negativo.

$n s^{* *}$ valor considerado não significativos quando comparado com o controle positivo.

DXR, doxorrubicina.

Fonte: Autoria própria

As larvas tratadas apenas com o óleo de copaíba nas concentrações de $0,5 \%, 1 \%$ e $2 \%$ apresentaram frequências de 0,24, 0,22 e 0,26 tumores por mosca, respectivamente. Analisando-se esses valores percebe-se que o óleo de copaíba, quando comparado com o controle negativo, não induziu um número significativo de tumores nas concentrações $0,5 \%$ e $1 \%$, entretanto, este número foi significativamente maior $(p \leq 0,05)$ na concentração $2 \%$. Sendo assim, pode-se dizer que o óleo de copaíba apresentou ação carcinogênica em Drosophila melanogaster na concentração $2 \%$.

Nos tratamentos com doxorrubicina associada a diferentes concentrações do óleo de copaíba $(0,5 \%, 1 \%$ e $2 \%)$ foram verificadas as frequências de 2,13; 1,94 e 1,24 tumores por mosca, respectivamente. Comparando-se estes valores ao controle positivo verifica-se um aumento significativo do número de tumores nas concentrações $0,5 \%$ e $1 \%$ ( $p \leq 0,05)$, o que não ocorreu na maior concentração testada (2\%). Pode-se dizer então, que o óleo de copaíba atuou como potencializador do efeito carcinogênico da doxorrubicina em Drosophila melanogaster nas concentrações $0,5 \%$ e $1 \%$.

\section{DISCUSSÃO}

Brito et al. (2010) também evidenciaram estimulação de crescimento tumoral pelo óleo de copaíba da espécie Copaifera officinalis em ratas com carcinoma de Walker 256. No estudo foi verificado crescimento tumoral de cerca de $70 \%$ quando utilizada a dose de $4,8 \mathrm{~mL} / \mathrm{kg}$ do óleo de copaíba. Os autores atribuíram os achados a uma possível ação imunossupressora da substância, o que justificaria também seu efeito anti-inflamatório.

Montes et al. (2009) chamam atenção especificamente para os efeitos do ácido caurenoico, um dos diterpenos presentes no óleo de copaíba. Revisão bibliográfica realizada pelos autores evidencia três estudos em que foi possível observar os efeitos citotóxicos da substância. Em um destes, realizado por Cavalcanti et al. (2006), ficou evidenciado ainda o efeito genotóxico do ácido caurenoico em altas doses, ou seja, sua capacidade de induzir danos ao DNA.

Tendo em vista o efeito genotóxico do ácido caure- noico, este, provavelmente, é um dos responsáveis pelo efeito carcinogênico evidenciado nas Drosophila melanogaster tratadas com óleo de copaíba na concentração de $2 \%$, sem associação com doxorrubicina. A menor ocorrência de tumores nas concentrações de 0,5\% e 1\% provavelmente se deve à menor concentração desse e outros compostos.

Quanto à ação moduladora observada sobre o efeito da doxorrubicina nas concentrações de 0,5\% e 1\%, é possível que tenha ocorrido sinergismo entre os efeitos genotóxicos do ácido caurenoico e da DXR, potencializando a carcinogênese. Alternativamente, pode-se considerar a possibilidade de que uma ou mais substâncias presentes no óleo de copaíba tenham efeito inibitório sobre enzimas do citocromo $\mathrm{P} 450$ responsáveis pela metabolização e inativação da doxorrubicina no organismo. É possível também que outros diterpenos e sesquiterpenos presentes no óleo de copaíba tenham contribuído para os efeitos carcinogênico e modulador do composto.

É notável a correlação inversamente proporcional entre o número de tumores induzidos pelo óleo de copaíba quando associado à doxorrubicina e a concentração do óleo utilizada, ou seja, o número de tumores foi substancialmente maior na menor concentração testada (0,5\%). A menor frequência de tumores na maior concentração do óleo testada (2\%) associada à doxorrubicina, provavelmente está relacionada com o efeito citotóxico causado pela substância, ou seja, na mais alta concentração, os erros induzidos no DNA são tão grosseiros que desencadeiam a via de apoptose.

A ativação da via apoptótica consiste em um processo ativo de colapso celular extremamente regulado e de grande eficiência, que requer a interação de inúmeros fatores. As alterações morfológicas observadas, como a condensação cromatínica, a fragmentação do DNA e a formação de corpos apoptóticos são consequência de uma cascata de eventos moleculares e bioquímicos geneticamente regulados. Uma vez desencadeados esses eventos, a célula sucumbe em sua própria destruição, caracterizando a apoptose (SILVA; NEPOMUCENO, 2011). 


\section{CONCLUSÃO}

O teste para detecção de clones de tumores epiteliais (warts) em Drosophila melanogaster permitiu identificar o potencial carcinogênico do óleo de copaíba (Copaifera officinalis L.) quando utilizado isoladamente na concentração $2 \%$. Além disso, demonstrou-se o efeito modulador do mesmo sobre a ação carcinogênica da doxorrubicina nas concentrações $0,5 \%$ e $1 \%$, uma vez que houve potencialização na frequência de tumores induzidos pela DXR.

Apesar dos resultados obtidos, não se pode deixar de considerar os trabalhos que mostraram diferentes implicações do óleo, evidenciando inclusive uma possível ação anticarcinogênica. Sendo assim, percebe-se a necessidade de estudos mais robustos utilizando o óleo de copaíba (Copaifera officinalis L.) e, se possível, com seus princípios isolados, utilizando diferentes metodologias e diferentes organismos testes.

\section{REFERÊNCIAS}

ALVES, E.M.; NEPOMUCENO, J.C. Avaliação do efeito anticarcinogênico do látex do avelós (Euphorbia tirucalli), por meio do teste para detecção de clones de tumor (warts) em Drosophila melanogaster. Perquirere, [S.I], v. 9, n. 2, p. 125-140, 2012.

ARAÚJO JUNIOR, F. A. et al. Efeito do óleo de copaíba nas aminotransferases de ratos submetidos à isquemia e reperfusão hepática com e sem pré-condicionamento isquêmico. Acta cir. bras., São Paulo, v. 20, n. 1, p. 93-99, 2005.

BASILE, A.C. et al. Anti-inflamatory activity of oleoresin from Brazilian Copaifera. J. Ethnopharmacol., Limerick, v. 22, p. 101-109, 1988.

BRASIL. Ministério da Saúde. Secretaria de Ciência, Tecnologia e Insumos Estratégicos. Departamento de Assistência Farmacêutica. Política Nacional de plantas medicinais e fitoterápicos. Brasília, DF, 2006. 60 p.

BRITO, N. M. B. et al. The effect of copaiba balsam on Walker 256 carcinoma inoculated into the vagina and uterine cervix of female rats. Acta cir. bras., São Paulo, v. 25, n. 2, p. 176-180, 2010.

CALIXTO, J. B. et al. Biological activity of plant extracts: novem analgesic drugs. Exp. Opin. Emerg. Drugs, [S.I], v. 6, n. 2, p. 261-279, 2001.

CAVALCANTI, B. C. et al. Genotoxicity evaluation of kaurenoic acid, a bioactive diterpenoid present in Copaiba oil. Food Chem. Toxicol., Oxford, v. 44, n. 3, p. 388-392, 2006.

COSTA-LOTUFO, L.V. et al. The cytotoxic and embryotoxic effects of kaurenoic acid, a diterpene isolated from Copaifera langsdorffii oleo-resin. Toxicon, Oxford, v. 40, n. 8, p. 1231-1234, 2002.

DRUMOND, M. R. S. et al. Estudo Comparativo in vitro da atividade antibacteriana de produtos fitoterápicos sobre bactérias cariogênicas. Pesqui. bras. odontopediatria e clín. integr., João Pessoa, v. 4, n. 1, p. 33-38, 2004.

FERNANDES, R.M.; PEREIRA, N.A.; PAULO, L.G. Anti-inflammatory activy of copaíba balsam (Copaifera cearensis, Huber). Rev. bras. farm., Rio de Janeiro, v. 73, n. 3, p. 53-56, 1992.
GIULIETTI, A. M. et al. Biodiversidade e conservação das plantas no Brasil. Megadiversidade, v. 1, n. 1, p. 52-61, 2005. Disponível em: <http://www. conservacao.org/publicacoes/ files /09_Giulietti_et_al.pdf >. Acesso em: 18 fev. 2015.

GUERRA, P.M.; NODARI, O.R. Biodiversidade: aspectos biológicos, geográficos, legais e éticos. In: SIMÕES, C. M. O. et al. Farmacognosia: da planta ao medicamento. 3. ed. Porto Alegre: UFRGS; Florianópolis: UFSC, 2001. p. 15.

JUSTICE, R.W. et al. The Drosophila tumor suppressor gene warts encodes a homolog o-f human myotonic dystrophy kinase and is required for the control of cell shape and proliferation. Genes \& Development, Cold Spring Harbor NY, v. 9, n. 5, p. 534-546, 1995.

MASSON, D. S. Atividades cicatrizante e antimicrobiana do óleo-resina de copaíba (Copaifera langsdorffii) em úlceras cutâneas. 2011. 215 f. Tese (Doutorado em Clínica Médica) - Faculdade de Medicina de Ribeirão Preto, Universidade de São Paulo, Ribeirão Preto, 2011.

MONTES, L. V. et al. Evidências para o uso da óleo-resina de copaíba na cicatrização de ferida - uma revisão sistemática. Natureza on line, Espírito Santo, v. 7, n. 2, p. 61-67, 2009. Disponível em: <http://www. naturezaonline.com.br/natureza/conteudo/pdf/02_monteslvetal_6167. pdf>. Acesso em: 24 abr. 2016.

OHSAKI, A. et al. The isolation and in vivo potent antitumor activity of clerodane diterpenoid from the oleoresin of the Brasilian medicinal plant, Copaifera langsdorffii Desf. Bioorg. Med. Chem Lett., Oxford, v. 4, n. 24, p. 2889-2892, 1994.

OLIVEIRA, E.C.P.; LAMEIRA, O.A.; ZOGHBI, M.G.B. Identificação da época de coleta do óleo-resina de copaíba (Copaifera spp.) no município de Moju, PA. Rev. bras. plantas. med., São Paulo, v. 8, n. 3, p. 14-23, 2006.

OLIVEIRA, L. A. R.; MACHADO, R. D.; RODRIGUES, A. J. L. Levantamento sobre o uso de plantas medicinais com a terapêutica anticâncer por pacientes da Unidade Oncológica de Anápolis. Rev. bras. plantas. med., São Paulo, v. 16, n. 2, p. 32-40, 2014

PAIVA, L.A. et al. Gastroprotective effect of Copaifera langsdorffii óleo-resin on experimental gastric ulcer models in rats. J. ethnopharmacol., Limerick, v. 62, n. 1, p. 73-78, 1998.

PIERI, F.A.; MUSSI, M.C.; MOREIRA, M.A.S. Óleo de copaíba (Copaifera sp.): histórico, extração, aplicações industriais e propriedades medicinais. Rev. bras. plantas. med., São Paulo, v. 11, n. 4, p. 465-472, 2009.

RIGAMONTE-AZEVEDO, O.C.; WADT, P.G.S.; WADT, L.H.O. Copaíba: ecologia e produção de óleo-resina. Rio Branco: EMBRAPA, 2004. 28 p.

SILVA, L.M.; NEPOMUCENO, J.C. Efeito modulador da polpa da graviola (Annona muricata) sobre a carcinogenicidade da mitomicina C, avaliado por meio do teste para detecção de clones de tumor (warts) em Drosophila melanogaster. Perquirere, [S.I], v. 1, n. 8, p. 80-94, 2011.

VEIGA JUNIOR, V.F.; PINTO, A.C. O gênero Copaifera L genus. Qim. nova, São Paulo, v. 25, n. 2, p. 273-286, 2002.

VEIGA JUNIOR, V.F.; PINTO, A.C.; MACIEL, M.A.M. Plantas medicinais: cura segura? Qui. nova, São Paulo, v. 28, n. 3, p. 519-528, 2005.

Submetido em: $12 / 04 / 2017$

Aceito em: 08/06/2017 